\title{
P113 The Forgotten Black Box of Blood Pressure: Error in Oscillometric Mean Arterial Pressure is Associated with Cuff Measurement Inaccuracy
}

\author{
Dean Picone ${ }^{1}$, Martin Schultz ${ }^{1}$, Matthew Armstrong ${ }^{1}$, Nathan Dwyer ${ }^{1,2}$, Philip Roberts-Thomson ${ }^{1,2}$, \\ Thomas Weber ${ }^{3}$, James Sharman ${ }^{2}$ \\ ${ }^{1}$ Menzies Institute for Medical Research, College of Health and Medicine, University of Tasmania, Australia \\ ${ }^{2}$ Royal Hobart Hospital, Hobart, Australia \\ ${ }^{3}$ Cardiology Department, Klinikum Wels-Grieskirchen, Wels, Austria
}

\section{ABSTRACT}

Background: Accurate measurement of cuff systolic BP (SBP) and diastolic BP (DBP) is contingent on the assumption of oscillometric MAP being accurate. If MAP is inaccurate this is likely to impact the accuracy of cuff SBP and DBP, but this has never been determined and was the aim of the study.

Methods: In five independent studies, MAP was measured by five unique cuff oscillometric BP devices at the same time as invasive aortic MAP (area under the waveform) by fluid-filled or solid-state catheters among 262 patients (61 \pm 11 years, $65 \%$ male) during coronary angiography. Cuff SBP and DBP were estimated by device-specific algorithms. Measurement errors were calculated as cuff - invasive aortic BP.

Results: For each BP device, MAP error was $-2.6 \pm 6.1,0.76 \pm 7.2,1.4 \pm 5.5,1.5 \pm 6.7$ and $7.1 \pm 9.2 \mathrm{mmHg}$. Corresponding SBP errors were $4.4 \pm 8.7,-0.8 \pm 11.2,-8.8 \pm 10.4,-0.9 \pm 10.5$ and $1.9 \pm 10.8 \mathrm{mmHg}$, whereas DBP errors were $8.8 \pm 5.1$, $2.0 \pm 7.4,6.7 \pm 7.3,10.3 \pm 9.0$ and $13.1 \pm 6.4 \mathrm{mmHg}$. There was a direct relationship between errors in oscillometric MAP and SBP error in four of the five devices ( $B$ range $=0.42$ to 1.04 ). However, MAP error was consistently related to DBP error in all devices ( $B$ range $=0.48$ to 0.97 ). Across all devices, absolute error in MAP $\geq 3 \mathrm{mmHg}$ corresponded to absolute error in SBP and DBP $\geq 5 \mathrm{mmHg}$ in $56-77 \%$ and $62-88 \%$ of patients.

Conclusion: Errors in oscillometric MAP are directly related to cuff SBP and DBP inaccuracy, but the magnitude of error is device-specific. Further work is required to understand algorithms used in oscillometric devices to improve cuff BP accuracy.

(c) 2019 Association for Research into Arterial Structure and Physiology. Publishing services by Atlantis Press International B.V. This is an open access article distributed under the CC BY-NC 4.0 license (http://creativecommons.org/licenses/by-nc/4.0/). 